\title{
Direct Numerical Simulations of High-Speed Turbulent Boundary Layers over Riblets
}

\author{
Lian Duan* \\ Missouri University of Science and Technology, Rolla, MO 65409 \\ Meelan M. Choudhari ${ }^{\dagger}$ \\ NASA Langley Research Center, Hampton, VA 23681
}

\begin{abstract}
Direct numerical simulations (DNS) of spatially developing turbulent boundary layers over riblets with a broad range of riblet spacings are conducted to investigate the effects of riblets on skin friction at high speeds. Zero-pressure gradient boundary layers under two flow conditions (Mach 2.5 with $T_{w} / T_{r}=1$ and Mach 7.2 with $T_{w} / T_{r}=0.5$ ) are considered. The DNS results show that the drag-reduction curve $\left(\Delta C_{f} / C_{f}\right.$ vs $\left.l_{g}^{+}\right)$at both supersonic speeds follows the trend of low-speed data and consists of a 'viscous' regime for small riblet size, a 'breakdown' regime with optimal drag reduction, and a 'drag-increasing' regime for larger riblet sizes. At $l_{g}^{+} \approx 10$ (corresponding to $s^{+} \approx 20$ for the current triangular riblets), drag reduction of approximately $7 \%$ is achieved at both Mach numbers, and confirms the observations of the few existing experiments under supersonic conditions. The Machnumber dependence of the drag-reduction curve occurs for riblet sizes that are larger than the optimal size, with smaller slopes of $\Delta C_{f} / C_{f}$ for larger freestream Mach numbers. The Reynolds analogy holds with $2 C_{h} / C_{f}$ approximately equal to that of flat plates for both drag-reducing and drag-increasing configurations.
\end{abstract}

\section{Nomenclature}

A surface area, $\mathrm{m}^{2}$

$C_{f} \quad$ Skin friction coefficient, dimensionless

$C_{h} \quad$ Stanton number, dimensionless

$C_{p}$ heat capacity at constant pressure, $\mathrm{J} /(\mathrm{K} \cdot \mathrm{kg})$

$C_{v}$ heat capacity at constant volume, $\mathrm{J} /(\mathrm{K} \cdot \mathrm{kg})$

$D$ Drag, $\mathrm{N}$

$h$ riblet height, $\mathrm{m}$

$L \quad$ Computational domain size, $\mathrm{m}$

$M \quad$ Mach number, dimensionless

$\operatorname{Pr} \quad$ Prandtl number, $P r=0.71$, dimensionless

$R \quad$ radius of riblet tip

$R e_{\theta} \quad$ Reynolds number based on momentum thickness and freestream viscosity, $R e_{\theta} \equiv \frac{\rho_{\infty} u_{\infty} \theta}{\mu_{\delta}}$, dimensionless

$R e_{\delta 2}$ Reynolds number based on momentum thickness and wall viscosity, $R e_{\delta_{2}} \equiv \frac{\rho_{\infty} u_{\infty} \theta}{\mu_{w}}$, dimensionless

$R e_{\tau}$ Reynolds number based on shear velocity and wall viscosity, $R e_{\tau} \equiv \frac{\rho_{w} u_{\tau} \delta}{\mu_{w}}$, dimensionless

$s \quad$ riblet spacing, $\mathrm{m}$

$T$ temperature, $\mathrm{K}$

$T_{r} \quad$ recovery temperature, $T_{r}=T_{\infty}\left(1+0.9 * \frac{\gamma-1}{2} M_{\infty}^{2}\right), \mathrm{K}$

$u \quad$ streamwise velocity, $\mathrm{m} / \mathrm{s}$

$u_{\tau} \quad$ friction velocity, $\mathrm{m} / \mathrm{s}$

$v \quad$ spanwise velocity, $\mathrm{m} / \mathrm{s}$

*Assistant Professor. Member, AIAA

${ }^{\dagger}$ Aerospace Technologist, Computational AeroSciences Branch, M.S. 128. Associate Fellow, AIAA 
$w \quad$ wall-normal velocity, $\mathrm{m} / \mathrm{s}$

$z_{\tau} \quad$ viscous length, $z_{\tau}=\nu_{w} / u_{\tau}, \mathrm{m}$

$\alpha \quad$ riblet ridge angle, degree

$\gamma \quad$ specific heat ratio, $\gamma=C_{p} / C_{v}$, dimensionless

$\delta \quad$ boundary layer thickness, $\mathrm{m}$

$\delta^{*} \quad$ displacement thickness, $\mathrm{m}$

$\kappa \quad$ thermal conductivity, $\kappa=\mu C_{p} / P r, \mathrm{~W} /(\mathrm{m} \cdot \mathrm{K})$

$\theta \quad$ momentum thickness, $\mathrm{m}$

$\mu \quad$ dynamic viscosity, $\mu=1.458 \times 10^{-6} \frac{T^{3 / 2}}{T+110.4}, \mathrm{~kg} /(\mathrm{m} \cdot \mathrm{s})$

$\nu \quad$ kinematic viscosity, $\nu=\mu / \rho, \mathrm{m}^{2} \cdot \mathrm{s}$

$\rho \quad$ density, $\mathrm{kg} / \mathrm{m}^{3}$

Subscripts

$f \quad$ quantities related to flat plate or clean surface

$i \quad$ inflow station for the domain of principal direct numerical simulation

$n \quad$ surface normal direction

$r \quad$ quantities related to riblets

rms root mean sqaure

$w \quad$ wall variables

$x \quad$ streamwise direction of the right-hand Cartesian coordinate

$y \quad$ spanwise direction of the right-hand Cartesian coordinate

$z \quad$ wall-normal direction of the right-hand Cartesian coordinate

$\infty \quad$ freestream variables

Superscripts

$+\quad$ inner wall units

$\overline{(\cdot)}$ averaged variables

$(\cdot)^{\prime}$ perturbation from averaged variable

\section{Introduction}

Riblets are micro-grooves aligned with the flow direction, and they are considered one of the premier techniques for reducing turbulent drag. ${ }^{1}$ Laboratory experiments and numerical simulations have shown that riblets of different geometries can reduce skin-friction drag by up to 10 percent in turbulent boundary layers (see reviews by Walsh, ${ }^{2}$ Choi, ${ }^{3}$ and Garcia-Mayoral \& Jimenez ${ }^{4}$ ). Moreover, riblets have been successfully applied to full aerodynamic configurations. ${ }^{5,6}$ For example, the flight tests of a commercial airplane (Airbus 320 ) with riblets over 70 percent of its surface imply an overall 2 percent drag reduction, based on the fuel savings obtained. ${ }^{7}$

The performance of riblets in reducing drag depends on their size. Typically, the riblet dimensions are normalized by viscous length scale, $z_{\tau}$, to account for the effect of Reynolds number. In the subsonic regime, both experiments and direct numerical simulations (DNS) show that a typical curve of drag reduction as a function of riblet spacing consists of a 'viscous' regime where the reduction in drag is proportional to the riblet size, a 'breakdown' regime where the linear dependence breaks down and a minimum drag is reached at some optimal spacing, and a 'drag-increasing' regime where riblets behave as the k-type roughness and induce a drag increase (Figure 1). ${ }^{8}$ The viscous regime exists in the limit of very small riblets when the riblet is within the viscous sublayer, and the slope of the drag curve in the viscous regime, $m_{s}$, can be well quantified by the Stokes theory. ${ }^{9}$ Within the breakdown regime, riblet effect saturates, and a minimum drag is reached for spacings $s_{o p t}^{+} \approx 10-20$. The optimal spacing $s_{o p t}^{+}$limits the maximum achievable drag reduction for a given riblet geometry. As the riblets get even larger, they become drag-increasing rather than drag-decreasing, and the slope of $\Delta \tau / \tau_{o}$ versus $s^{+}$curve within the drag-increasing regime determines the robustness of a particular riblet geometry in reducing drag. To design riblets with optimal peak performance and robustness, it is critical to identify flow parameters that could influence the extent of each regime and understand the mechanisms that control the transition among the different riblet-spacing regimes. GarciaMayoral and Jimenez ${ }^{4}$ provided a recent review of available experiments and theoretical studies of the different drag-reduction regimes, as well as the physical mechanisms proposed in the literature both for the viscous regime and for its breakdown. They showed that the groove cross section, $A_{g}$ (or $l_{g}=\sqrt{A_{g}}$ ), which accounts for both riblet spacing, $s$, and depth, $h$ (Figure 2), provides a better collapse of the drag- 
reduction curve for riblets of different geometries than the riblet spacing, $s$, with the optimal drag reduction being $l_{g, \text { opt }}^{+} \approx 10.7 \pm 1$. Garcia-Mayoral and Jimenez ${ }^{4,10}$ also hypothesized that the viscous breakdown is caused by the appearance of long spanwise rollers below $z^{+} \approx 20$. The spanwise rollers are spanwise coherent structures that extend several inter-riblet spacings and that are developed from a two-dimensional Kelvin-Helmholtz-like instability of the mean streamwise flow.

Most previous studies on the different drag-reduction regimes of riblets are limited to incompressible flows, and, typically, channel flows. Very limited riblet data at supersonic speeds are available. The few data include wind tunnel investigations at Mach 2.97 by Robinson, ${ }^{11}$ Mach 1.5 by Gaudet, ${ }^{12}$ Mach 1.6, 2.0, and 2.5 by Coustols and Cousteix, ${ }^{13}$ as well as flight tests at Mach $1.2-1.6$ by Zuniga et al. ${ }^{14}$ These studies tested riblets with $s^{+} \approx 9-15$ and reported skin friction drag reduction of $\Delta C_{f} / C_{f} \approx 4-15 \%$. However, none of the above studies have provided a simultaneous measurement of skin-friction drag and near-wall turbulent flow field to allow for a complete characterization of the flow features in each regime and the mechanisms that control transition among regimes. Duan and Choudhari ${ }^{15}$ conducted DNS of high-speed turbulent boundary layers to examine the effect of riblets on skin friction under two flow conditions (Mach 2.5 with $T_{w} / T_{r}=1$ and Mach 7.2 with $T_{w} / T_{r}=0.5$ ). They considered symmetric $\mathrm{V}$-groove riblets with two rib spacings $\left(s^{+} \approx 20\right.$ and $\left.s^{+} \approx 40\right)$. Unlike most of the previous DNS, , $16-18$ which focused on incompressible channel flows, they examined riblets in external, spatially-developing flows without any assumption of streamwise periodicity. An identical inflow was used for simulations with different riblet spacings to provide a cleaner setting to compare drag. Their DNS results were consistent with the few existing experimental observations and showed that a drag reduction of approximately $7 \%$ can be achieved for riblets with proper spacing at high speeds. Their study also helped to establish a correspondence between riblets in an incompressible channel flow and those over a flat plate, especially under a supersonic and hypersonic free stream. However, with most of the work by Duan and Choudhari ${ }^{15}$ focused on comparing the turbulence statistics and flow visualizations between drag-reducing and drag-increasing configurations, a complete characterization of flow features across different drag-reduction regimes for high-speed flows is still lacking.

One of the primary objectives of the current study is to extend the work by Duan and Choudhari ${ }^{15}$ to obtain a DNS dataset of supersonic external flows over riblet surfaces across all the riblet-spacing regimes ('viscous', 'breakdown', and 'drag-increasing'), and provide detailed data for understanding drag-reduction mechanisms and developing reduced-order models for drag prediction. The effects of compressibility on the drag-reduction curve is explored and the similarities and differences in the drag reduction mechanisms between incompressible and high-speed boundary layers are investigated.

The paper is structured as follows. The flow conditions and numerical methods are outlined in Section II. Section III presents results pertaining to the effects of riblets on skin friction at high speeds, including the drag-reduction curve and drag-reduction mechanisms, followed by a summary in Section IV.

\section{Flow Conditions and Numerical Methodology}

The boundary layer edge conditions and wall parameters for the DNS are given in Table 1, which provides freestream Mach number, density, and temperature $\left(M_{\infty}, \rho_{\infty}\right.$, and $T_{\infty}$, respectively) as well as the boundary layer thickness and various Reynolds numbers at the inlet of the domain. We conduct DNS for two spatially developing boundary layers in the high-speed regime. One is at Mach 2.5, which is relevant to supersonic transports, and the other is at Mach 7.2, which falls within the hypersonic regime.

Table 1. Bounday layer edge and wall parameters for the DNS.

\begin{tabular}{ccccccccc}
\hline$M_{\infty}$ & $\rho_{\infty}\left(\mathrm{kg} / \mathrm{m}^{3}\right)$ & $T_{\infty}(\mathrm{K})$ & $T_{w}(\mathrm{~K})$ & $T_{w} / T_{r}$ & $\delta_{i}(\mathrm{~mm})$ & $R e_{\theta}$ & $R e_{\tau}$ & $R e_{\delta 2}$ \\
\hline 2.5 & 0.1 & 270.4 & 568.0 & 1.0 & 4.00 & $1,777.5$ & 340.4 & $1,038.9$ \\
7.2 & 0.07 & 65.8 & 352.0 & 0.5 & 9.06 & $6,536.3$ & 392.4 & $1,372.3$ \\
\hline
\end{tabular}

The compressible Navier-Stokes equations are solved in generalized curvilinear coordinates. The working fluid is assumed to be an ideal gas with a linear (i.e., Newtonian) stress-strain relation. The Fourier law is used to compute the heat flux terms. A 7th order weighted essentially non-oscillatory (WENO) scheme ${ }^{19}$ is used to compute the convective flux terms. This particular WENO algorithm combines a high order of accuracy with low dissipation and high bandwidth, which is desirable for time accurate simulations of 
compressible turbulence. The resolution properties of this scheme are documented in several references. ${ }^{20,21}$ For the viscous flux terms, a 4th order central difference scheme is used. The 3rd order low storage RungeKutta scheme by Williamson ${ }^{22}$ is used for time integration.

To enable the simulation of a spatially evolving boundary layer, we conduct an independent auxiliary DNS of a spatially developing boundary layer which provides the inflow boundary condition for the principal DNS over the domain with riblets (Figure 3). The single auxiliary DNS is used to provide an identical inflow for the downstream simulations with and without riblets, thus isolating the influence of riblets on skin friction drag. To minimize the extent of upstream/downstream influence due to the surface variation, the riblets begin at a distance downstream of the inlet of the principal DNS and the grids consist of a smooth streamwise transition from the clean surface to the riblet surface (Figure 4a). ${ }^{15}$ On the wall, no-slip conditions are applied for the three velocity components and an isothermal condition is used for the temperature, with the wall temperature listed in Table 1 . The wall temperature is prescribed to be nearly the recovery temperature, $T_{r}$, for the Mach 2.5 cases and $0.5 T_{r}$ for the Mach 7.2 cases. At the top and outlet boundaries, unsteady non-reflecting boundary conditions based on Thompson ${ }^{23}$ are imposed. Periodic boundary conditions are used in the spanwise direction.

For each freestream condition, simulations with different surface topologies have been undertaken with riblet spacing the primary parameter as outlined in Table 2. To our knowledge, this is the first time that such a systematic parameter sweep has been undertaken for numerical riblets under supersonic speeds. For each riblet simulation, triangular riblets with ridge angle of $45^{\circ}$ are used. To improve smoothness of the grids and avoid singularities when evaluating the Jacobian, the riblet tips and valleys are slightly rounded with with radius $R / s \approx 4 \%$. The extent of rounding is within a range where no significant degradation in riblet performances is expected. ${ }^{24,25}$ More importantly, as discussed by Garcia-Mayoral and Jimenez, ${ }^{10}$ such a tip-rounding should not significantly affect the viscous breakdown, because they barely modify the groove cross section. A close-up view of riblet geometry and near-wall grids has been sketched in Figure $4 \mathrm{~b}$.

For DNS cases with riblets, the riblets begin at a distance $x \approx 4.8 \delta_{i}$ downstream of the inlet of the principal DNS, while upstream of this position the surface remains clean to guarantee a nearly unperturbed flow at the inlet. Moreover, the grids consist of a smooth streamwise transition from the clean surface to the riblet surface to minimize the extent of upstream/downstream influence due to the surface variation. The streamwise length for the transitional zone is approximately four times that of the riblet height, $h$, with a finer spacing across the transition length, as is shown in Figure 4a. The streamwise grid is uniform on both sides of the transitional zone. The riblet zone has a streamwise extent of more than $10 \delta_{i}$. The spanwise grids are clustered near the riblet tips and coarsened near riblet valleys, with 32 grid points on the surface of each riblet. The spanwise extent of the domain is approximately 400 wall units. Such a spanwise extent is significantly larger than the values used by Choi and Moin ${ }^{16}$ and comparable with those used by Garcia-Mayoral and Jimenez. ${ }^{4}$

\section{Results}

In this section, we present DNS results based on the cases listed in Table 2. The effects of riblets on skin-friction drag and other turbulence statistics are examined, and the comparison of flow physics among different Mach-number cases is conducted. Statistics are collected near a streamwise location of $x_{a} \approx 18 \delta_{i}$ for the Mach 2.5 cases and $x_{a} \approx 15$ for the Mach 7.2 cases, which is significantly downstream of the beginning of the riblet surface to avoid any significant effects due to surface transition. To obtain statistics, averages are calculated over time, the same spanwise locations over different riblets, and a streamwise window of $\left[x_{a}-0.8 \delta_{i}, x_{a}+0.8 \delta_{i}\right]$. The time average spans more than $150 \delta_{i} / u_{\infty}$. We use an overbar to indicate an averaged quantity and a prime to indicate perturbations from this average. Averages are calculated over time, the same spanwise locations over different riblets, and the streamwise window. Reducing the number of flow fields for the ensemble averaging by one third or increasing the length of streamwise window by eight times result in less than $0.5 \%$ difference in viscous drag. The origin of the $z$ coordinate corresponds to the wall location for clean surfaces. For cases with riblets, the riblets are mounted on top of the otherwise clean surface with $z=0$ corresponding to the riblet valley. This computational setup mimics the practical applications wherein riblets are molded into a lightweight plastic film with an adhesive backing and pressed into place on the surface of a vehicle. The influence of the surface transition from clean surface to riblet surface has been assessed by varying the streamwise transitional length, and less than $0.5 \%$ difference in drag is observed at the downstream location where statistics are collected. 
Table 2. Simulation parameters for the direct numerical simulations of turbulent flows over riblets. The value of $\Delta x$ corresponds to that in the uniform region (outside of the transitional zone).

\begin{tabular}{lcccccccccc}
\hline Case & $s^{+}$ & $l_{g}^{+}$ & $N_{\text {riblet }}$ & $L_{x} / \delta_{i}$ & $L_{y} / \delta_{i}$ & $L_{z} / \delta_{i}$ & $\Delta x^{+}$ & $\Delta y^{+}$ & $\Delta z_{\text {min }}^{+}$ & $N_{x} \times N_{y} \times N_{z}$ \\
\hline M25Clean & NA & NA & NA & 20.4 & 1.6 & 11.3 & 10.3 & 1.34 & 0.28 & $528 \times 320 \times 120$ \\
M25s10 & 8.9 & 4.5 & 40 & 20.4 & 1.6 & 11.3 & 10.3 & $0.13-0.51$ & $0.20-0.27$ & $528 \times 1280 \times 120$ \\
M25s20 & 18.2 & 9.1 & 20 & 20.4 & 1.6 & 11.3 & 10.3 & $0.27-1.02$ & $0.20-0.27$ & $528 \times 640 \times 120$ \\
M25s30 & 27.6 & 13.8 & 14 & 20.4 & 1.6 & 11.3 & 10.3 & $0.39-1.53$ & $0.20-0.27$ & $528 \times 448 \times 120$ \\
M25s40 & 37.3 & 18.6 & 10 & 20.4 & 1.6 & 11.3 & 10.3 & $0.52-2.04$ & $0.13-0.28$ & $528 \times 320 \times 120$ \\
M25s50 & 47.6 & 23.8 & 8 & 20.4 & 1.6 & 11.3 & 10.3 & $0.65-2.55$ & $0.13-0.28$ & $528 \times 256 \times 120$ \\
M25s60 & 58.2 & 29.1 & 7 & 20.4 & 1.6 & 11.3 & 10.3 & $0.78-3.06$ & $0.13-0.28$ & $528 \times 224 \times 120$ \\
M72Clean & NA & NA & NA & 16.8 & 1.1 & 14.5 & 5.8 & 1.18 & 0.28 & $960 \times 320 \times 120$ \\
M72s20 & 20.8 & 10.4 & 20 & 16.8 & 1.1 & 14.5 & 5.8 & $0.16-1.29$ & $0.19-0.52$ & $960 \times 640 \times 120$ \\
M72s30 & 32.1 & 16.0 & 14 & 16.8 & 1.1 & 14.5 & 5.8 & $0.24-1.93$ & $0.19-0.57$ & $960 \times 448 \times 120$ \\
M72s40 & 43.8 & 21.9 & 10 & 16.8 & 1.1 & 14.5 & 5.8 & $0.32-2.64$ & $0.19-0.62$ & $960 \times 320 \times 120$ \\
M72s50 & 56.5 & 28.2 & 8 & 16.8 & 1.1 & 14.5 & 5.8 & $0.45-2.95$ & $0.18-0.62$ & $960 \times 256 \times 120$ \\
M72s60 & 59.9 & 34.9 & 7 & 16.8 & 1.1 & 14.5 & 5.8 & $0.64-3.86$ & $0.18-0.62$ & $960 \times 224 \times 120$ \\
\hline
\end{tabular}

\section{A. Drag Measurement}

The total skin-friction drag, $D$, is obtained as the integral of the product of the wall shear stress and the differential wetted area

$$
D=\int_{A_{r}} \mu_{w}\left(\frac{\partial u}{\partial n}\right)_{w} d A_{r}=\tau_{w}^{*} A_{f}=\mu_{w}\left(\frac{\partial u}{\partial n}\right)_{f}^{*} A_{f}
$$

where $A_{r}$ denotes the wetted area of a riblet surface and $A_{f}=A_{r} \cos (\alpha)=L_{x} \times L_{y}$ is its corresponding clean-surface area (see Figure 5).

The skin friction coefficient, $C_{f}$, is defined as

$$
C_{f}=\frac{\bar{\tau}_{w}^{*}}{\frac{1}{2} \rho_{\infty} u_{\infty}^{2}}
$$

Figure 6 shows typical time history of the plane-averaged skin-friction coefficient at $x / \delta_{i}=18$ after the initial transient for the Mach 2.5 cases. Consistent with the results at low speeds (Figure 1), the performance of riblets is a function of riblet size and there exist a drag-reducing regime and a drag-increasing regime.

Figure 7 further plots the drag-reduction curve, $\Delta C_{f} / C_{f}$ (time and space-averaged skin-friction variation relative to the clean surface), as a function of riblet size, $l_{g}^{+}=\left(A_{g}^{+}\right)^{1 / 2}$, for the various freestream Mach numbers. For reference, the envelopes of low-speed experimental results compiled by Garcia-Mayoral and Jimenez ${ }^{4}$ are included. Note that $l_{g}^{+}$rather than $s^{+}$is used to characterize the riblet size following the definition introduced by Garcia-Mayoral and Jimenez. ${ }^{4,10}$ Such a choice will facilitate the comparison with the low-speed data, since $l_{g}^{+}$has been shown to result in a better collapse of data at low speeds for riblets of different geometries. ${ }^{4,10}$ Similar to the low-speed counterpart, the drag reduction for both supersonic Mach numbers attains its maximum at $l_{g}^{+} \approx 10$, with a maximum drag reduction of approximately $7 \%$. The achieved drag reduction at both supersonic Mach numbers confirms the observations of the few existing experiments under supersonic conditions. ${ }^{12-14}$ The similarity in the amount of drag reduction at $l_{g}^{+} \approx 10$ among various Mach number cases may indicate that the dynamics of riblet flows in the breakdown regime is not significantly modified by compressibility effects up to a Mach number of 7.2.

The Mach-number dependence of the drag-reduction curve seems to occur for riblet sizes that are larger than the optimal size $l_{g, o p t}^{+} \approx 10$. As the freestream Mach number increases, the slope of $\Delta C_{f} / C_{f}$ at $l_{g}^{+}>l_{g, \text { opt }}^{+}$, which determines the robustness of a particular riblet geometry in reducing drag, reduces. As a result, the optimal drag reduction deteriorates at a lower rate and the drag-reducing regime persists up to a larger riblet size for the higher Mach number case. 
It should be noted that the above observations are only made based on the current data set. Additional data points in the vicinity of the maximum-drag-reduction location are necessary to confirm that $l_{g, \text { opt }}^{+}$stays approximately around 10, especially for the Mach 7.2 case, for which there is no data between $0<l_{g}^{+}<10.4$ within the current data set. Also, the extent of the 'viscous' regime and the exact location where the dragreduction curve begins to deviate from a linear relation cannot be fully characterized based on the current DNS datasets alone and needs to be determined via additional DNS together with Stokes calculations. ${ }^{9}$

\section{B. Near-wall Turbulence Structures and Drag Reduction Mechanisms}

In this subsection, near-wall turbulence structures are investigated in order to identify the similarities and differences in the mechanism by which riblets reduce drag between incompressible and supersonic flows.

In a recent DNS study of riblets for incompressible flows, Garcia-Mayoral and Jimenez ${ }^{4}$ found that the viscous breakdown is caused by the appearance of long spanwise vortical structures (referred to as spanwise rollers following Garcia-Mayoral and Jimenez) below $\left(z-z_{\text {tip }}\right)^{+} \approx 20$ with typical streamwise wavelengths $\lambda_{x}^{+} \approx 150$ that are developed from a two-dimensional Kelvin-Helmholtz-like instability of the mean streamwise flow. Figure $8 \mathrm{a}$ and Figure $8 \mathrm{~b}$ plot the instantaneous wall-normal velocity at $\left(z-z_{\text {tip }}\right) \approx 3$ to visualize the near-wall spanwise rollers for both supersonic Mach-number cases at $l_{g}^{+} \approx 10$. The spanwise rollers that are perpendicular to the riblets appear for both Mach-number cases (e.g., at $x^{+} \approx 1,200$ for the Mach 2.5 case), although it is much more obvious for the Mach 7.2 case. The existence of the spanwise coherent structures may indicate a connection between the spanwise rollers and the viscous breakdown at high speeds, as in the low-speed flows.

In terms of the drag-increase regime (corresponding to $l_{g}^{+}>l_{g, o p t}^{+}$), Choi and Moin ${ }^{16}$ argued that the deterioration of drag reduction for large riblet sizes is caused by the lodging (penetration) of the streamwise vortices inside the grooves so that a larger wetted area is exposed to the high-speed downwash induced by the streamwise vortices, resulting in a larger region of high shear rate. There exist similar streamwise vortices for high-speed flows, as visualized in Figure 9, and the characteristics of these vortices vary with the freestream Mach number and wall temperature. ${ }^{26,27}$ To estimate the average size and location of the near-wall streamwise vortices, Figure 10 plots the root-mean-square (RMS) of streamwise vorticity, $\omega_{x, r m s}^{+}$, as a function of the wall-normal distance for Cases M25Clean and M72Clean. For both supersonic Mach numbers, $\omega_{x, r m s}^{+}$shows a local minimum at $z_{\text {min }}^{+} \approx 5$ and attains a local maximum at $z_{\text {max }}^{+} \approx 20$, with $z_{\max }^{+}=17.4$ and $z_{\min }^{+}=4.5$ for Mach 2.5 and $z_{\max }^{+}=23.2$ and $z_{\min }^{+}=4.9$ for Mach 7.2. According to the streamwise vortex model proposed by Kim et al. ${ }^{28}$ (Figure 11), the local maximum corresponds to the average location of the center of the streamwise vortex, and the local minimum corresponds to the average location of the edge of the vortex. The larger values of $z_{\max }^{+}$and $\left(z_{\max }^{+}-z_{\min }^{+}\right)$for the Mach 7.2 case indicate that the streamwise vortices for the Mach 7.2 cases are of larger size and located farther from the wall compared with the Mach 2.5 case. As a result, for a given groove size, it is less likely for these vortices to lodge within or penetrate into the grooves, as shown in Figure 12. This may explain why the drag increase is less rapid for the Mach 7.2 case than the Mach 2.5 case for $l_{g}^{+}>l_{g, o p t}^{+}$.

\section{Surface Heat Flux}

The design of effective vehicle surfaces that minimizes surface heat flux is of paramount importance for preventing overheating of hypersonic vehicles. Thus, one of the most important factors that determine the applicability of riblets to hypersonic vehicles is their influence on surface heat flux. Although heat-transfer management using riblets has been studied extensively for low-speed flows, there is some controversy about their effectiveness ${ }^{29-31}$ and very few studies exist for high-speed flows. In this subsection, the effect of riblets on surface heat flux is investigated for the Mach 7.2 case, which has a wall temperature of $T_{w} / T_{r}=0.5$.

Similar to the total skin-friction drag, the total surface heat flux, $Q$, over a riblet surface, $A_{r}$, can be obtained as

$$
Q=\int_{A_{r}} \kappa_{w}\left(\frac{\partial T}{\partial n}\right)_{w} d A_{r}=q_{w}^{*} A_{f}=\kappa_{w}\left(\frac{\partial T}{\partial n}\right)_{f}^{*} A_{f}
$$

The Stanton number $C_{h}$ are defined as

$$
C_{h}=\frac{\bar{q}_{w}^{*}}{\rho_{\infty} u_{\infty} C_{p}\left(T_{r}-T_{w}\right)}
$$


and the Reynolds analogy factor $R A$ is defined as

$$
R A=\frac{2 C_{h}}{C_{f}}
$$

Figure 13a shows the variation in Stanton number, $C_{h}$, as a function of riblet size, $l_{g}^{+}$, for the Mach 7.2 case. Similar to the skin-friction drag, the surface heat flux reduces for small riblet sizes and increases for large riblets, with the maximum heat reduction at $l_{g}^{+} \approx 10$. The fact that a drag-reducing riblet surface also reduces surface heat flux is a feature that is desired in hypersonic applications, even if the reduction in heat transfer is modest.

For drag-reducing and drag-increasing riblet surfaces, the variation in heat transfer is larger than that in viscous drag with the Reynolds analogy factor $1.16<R A<1.19$ (Figure 13b). The Reynolds analogy factor for riblets assumes similar values as those for hypersonic flat-plate boundary layers. For comparison, the experimental data reviewed by Hopkins and Inouye ${ }^{32}$ give $1.1<R A<1.3$ for boundary layers with $T_{w} / T_{r}>0.3 . R A \approx 1$ indicates the validity of Reynolds analogy for riblet surfaces, at least for those with $l_{g}^{+}<30$.

The relatively larger variation in heat transfer than that in viscous drag and the validity of Reynolds analogy across a wide range of riblet sizes is consistent with the findings by Benhalilou and Kasagi ${ }^{33}$ and Stalio and Nobile ${ }^{31}$ for low-speed boundary layers. However, given that the temperature is only a passive scalar for low-speed flows but is tightly coupled with the velocity field for high-speed flows, it may be difficult to draw any direct analogy between the two speed regimes.

\section{Summary}

This paper presented direct numerical simulations of turbulent boundary layers over triangular riblets with a ridge angle of $45^{\circ}$ at Mach 2.5 and Mach 7.2. The DNS results show that the drag-reduction curve $\left(\Delta C_{f} / C_{f}\right.$ vs riblet size $)$ at both supersonic speeds follows the trend of low-speed data and consists of a 'viscous' regime for small riblet size, a 'breakdown' regime with optimal drag reduction, and a 'dragincreasing' regime for larger riblet sizes. At $l_{g}^{+} \approx 10$ (corresponding to $s^{+} \approx 20$ for the current triangular riblets), drag reduction of approximately $7 \%$ is achieved at both Mach numbers, and confirm the observations of the few existing experiments under supersonic conditions. The drag-reduction curve as a function of riblet spacing displays a noticeable dependence on the flow Mach number, with a weaker rate of reduction in drag benefit at the higher Mach number.

In terms of drag-reduction mechanisms, the DNS data show the existence of spanwise coherent vortical structures at $l_{g}^{+} \approx 10$, similar to the finding by Garcia-Mayoral and Jimenez. ${ }^{4}$ The reduction in the slope of $\Delta C_{f} / C_{f}$ for $l_{g}^{+}>l_{g, \text { opt }}^{+}$at the larger Mach number appears to be related to the location of streamwise vortical structures relative to the riblet grooves.

At Mach 7.2 with $T_{w} / T_{r}=0.5$, it is found that the Reynolds analogy holds with $1.16<R A=2 C_{h} / C_{f}<$ 1.19 for both drag-reducing and drag-increasing riblet surfaces.

Future work will target additional computations to determine the optimum riblet spacing at the higher Mach number as well as to provide a better characterization of the extent of the 'viscous' (i.e., linear) portion of the drag reduction curve at smaller riblet spacings.

\section{Acknowledgments}

This material is based on the work supported by National Aeronautics and Space Administration, Langley Research Center under the Research Cooperative Agreement No. NNL09AA00A awarded to the National Institute of Aerospace. The authors would like to thank Prof. Pino Martín of the University of Maryland for providing the original code which has been modified for the current study. The simulations have been conducted using the Pleiades Supercomputer of the NASA Advanced Supercomputing (NAS) Division and the Extreme Science and Engineering Discovery Environment (XSEDE), which is supported by National Science Foundation grant number OCI-1053575. 


\section{References}

${ }^{1}$ Bushnell, D. M. and McGinley, C. B., "Turbulence Control in Wall Flows," Annual Review of Fluid Mechanics, Vol. 21, No. 1, Jan. 1989, pp. 1-20.

${ }^{2}$ Walsh, M. J., "Riblets," Progress in Astronautics and Aeronautics, Washington, DC, AIAA, 1990, pp. $203-261$.

${ }^{3}$ Choi, K.-S., "European Drag-Reduction Research — Recent Developments and Current Status," Fluid Dyn. Res., Vol. 26, 2000, pp. 325-335.

${ }^{4}$ Garcia-Mayoral, R. and Jimenez, J., "Hydrodynamic Stability and Breakdown of the Viscous Regime over Riblets," Journal of Fluid Mechanics, Vol. 678, 2011, pp. 317-347.

${ }^{5}$ Viswanath, P. R., "Aircraft Viscous Drag Reduction Using Riblets," Progress in Aerospace Sciences, Vol. 38, No. 6-7, Aug. 2002, pp. 571-600.

${ }^{6}$ Bushnell, D. M., "Aircraft Drag Reductiona Review," Proc. Inst. Mech. Eng., Vol. 217, 2003, pp. 1-18.

${ }^{7}$ Szodruch, J., "Viscous Drag Reduction on Transport Aircraft," AIAA Paper 1991-0685, 1991.

${ }^{8}$ Bechert, D. W., Bruse, M., Hage, W., Hoeven, J., and Hoppe, G., "Experiments on Drag-Reducing Surfaces and Their Optimization with an Adjustable Geometry," Journal of Fluid Mechanics, Vol. 338, 1997, pp. 59-87.

${ }^{9}$ Luchini, P., Manzo, F., and Pozzi, A., "Resistance of a Grooved Surface to Parallel Flow and Cross-Flow," Journal of Fluid Mechanics, Vol. 228, 1991, pp. 87-109.

${ }^{10}$ Garcia-Mayoral, R. and Jimenez, J., "Drag Reduction by Riblets," Phil. Trans. R. Soc. A, Vol. 369, 2011, pp. $1412-1427$.

${ }^{11}$ Robinson, S. K., "Effects of Riblets on Turbulence in a Supersonic Boundary Layer," AIAA Paper 88-2526, 1988.

${ }^{12}$ Gaudet, L., "Properties of Riblets at Supersonic Speed," Applied Scientific Research, Vol. 46, No. 3, July 1989, pp. 245254.

${ }^{13}$ Coustols, E. and Cousteix, J., "Performances of Riblets in the Supersonic Regime," AIAA Journal, Vol. 32, No. 2, 1994, pp. 431-433.

${ }^{14}$ Zuniga, F. A., Anderson, B. T., and Bertelrud, A., "Flight Test Results of Riblets at Supersonic Speeds," NASA Technical Paper 4387, 1992.

${ }^{15}$ Duan, L. and Choudhari, M. M., "Effects of Riblets on Skin Friction and Heat Transfer in High-Speed Turbulent Boundary Layers," AIAA Paper 2012-1108, 2012.

${ }^{16}$ Choi, H., Moin, P., and Kim, J., "Direct Numerical Simulation of Turbulent Flow over Riblets," Journal of Fluid Mechanics, Vol. 255, 1993, pp. 503-539.

${ }^{17} \mathrm{Chu}$, D. C. and Karniadakis, G. E., "A Direct Numerical Simulation of Laminar and Turbulent Flow over Riblet-Mounted Surfaces," Journal of Fluid Mechanics, Vol. 250, 1993, pp. 1-42.

${ }^{18}$ Goldstein, D., Handler, R., and Sirovich, L., "Direct Numerical Simulation of Turbulent Flow over a Modelled Riblet Covered Surface," Journal of Fluid Mechanics, Vol. 302, 1995, pp. 333-376.

${ }^{19}$ Jiang, G. and Shu, C., "Efficient Implementation of Weighted ENO Schemes," Journal of Computational Physics, Vol. 126, No. 1, June 1996, pp. 202-228.

${ }^{20}$ Martín, M. P., Taylor, E. M., Wu, M., and Weirs, V. G., "A Bandwidth-Optimized WENO Scheme for the Direct Numerical Simulation of Compressible Turbulence," Journal of Computational Physics, Vol. 220, No. 1, 2006, pp. 270-289.

${ }^{21}$ Taylor, E. M., Wu, M., and Martín, M. P., "Optimization of nonlinear error sources for weighted non-oscillatory methods in direct numerical simulations of compressible turbulence," Journal of Computational Physics, Vol. 223, 2006, pp. 384-397.

${ }^{22}$ Williamson, J., "Low-Storage Runge-Kutta Schemes," Journal of Computational Physics, Vol. 35, No. 1, 1980 , pp. 48-56.

${ }^{23}$ Thompson, K. W., "Time Dependent Boundary Conditions for Hyperbolic Systems," Journal of Computational Physics, Vol. 68, No. 1, Jan. 1987, pp. 1-24.

${ }^{24}$ Walsh, M. J. and Lindemann, A. M., "Optimization and Application of Riblets for Turbulent Drag Reduction," AIAA Paper 84-0347, 1984.

${ }^{25}$ Walsh, M. J., "Effect of Detailed Surface Geometry on Riblet Drag Reduction Performance," Journal of Aircraft, Vol. 27, No. 6, 1990, pp. 572-573.

${ }^{26}$ Duan, L., Beekman, I., and Martín, M. P., "Direct Numerical Simulation of Hypersonic Turbulent Boundary Layers. Part 2: Effect of Wall Temperature," Journal of Fluid Mechanics, Vol. 655, 2010, pp. 419-445.

${ }^{27}$ Duan, L., Beekman, I., and Martín, M. P., "Direct Numerical Simulation of Hypersonic Turbulent Boundary Layers. Part 3: Effect of Mach Number," Journal of Fluid Mechanics, Vol. 672, 2011, pp. 245-267.

${ }^{28}$ Kim, J., Moin, P., and Moser, R., "Turbulence Statistics in Fully Developed Channel Flow at Low Reynolds Number," Journal of Fluid Mechanics, Vol. 177, 1987, pp. 133-166.

${ }^{29}$ Lindemann, A. M., "Turbulent Reynolds Analogy Factors for Nonplanar Surface Microgeometries," Journal of Spacescraft, Vol. 22, No. 5, 1985, pp. 581-582.

${ }^{30}$ Choi, K. S. and Orchard, D. M., "Turbulence Management Using Riblets for Heat and Momentum Transfer," Experimental Thermal and Fluid Science, Vol. 15, 1997, pp. 109-124.

${ }^{31}$ Stalio, E. and Nobile, E., "Direct Numerical Simulation of Heat Transfer over Riblets," International Journal of Heat and Fluid Flow, Vol. 24, 2003, pp. 356-371.

${ }^{32}$ Hopkins, E. J. and Inouye, M., "Evaluation of Theories for Predicting Turbulent Skin Friction and Heat Transfer on Flat Plates at Supersonic and Hypersonic Mach Mumbers," AIAA Journal, Vol. 9, No. 6, 1971, pp. 993-1003.

${ }^{33}$ Benhalilou, M. and Kasagi, N., "Numerical prediction of heat and momentum transfer over micro-grooved surface with a nonlinear $k-\epsilon$ model," International Journal of Heat and Mass Transfer, Vol. 42, No. 14, 1999, pp. 2525-2541.

${ }^{34}$ Robert, J. P., "Drag Reduction: An Industrial Challenge," AGARD-R-796, March 1992. 


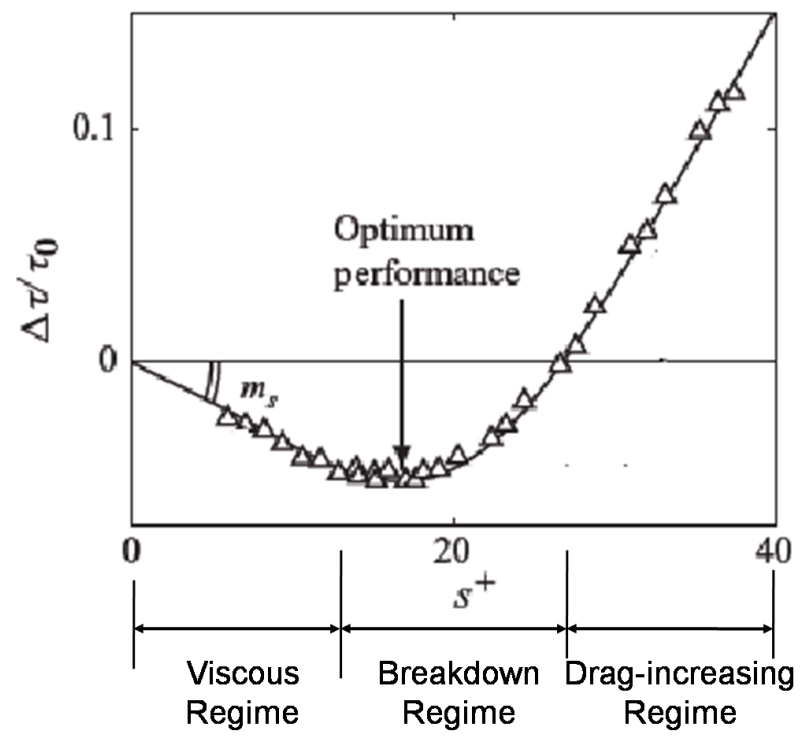

Figure 1. Definition of the drag-reduction regimes observed over triangular riblets with $60^{\circ}$ tip angle, as a function of the peak-to-peak distance $s^{+}$. Adapted from Bechert et al. ${ }^{8}$

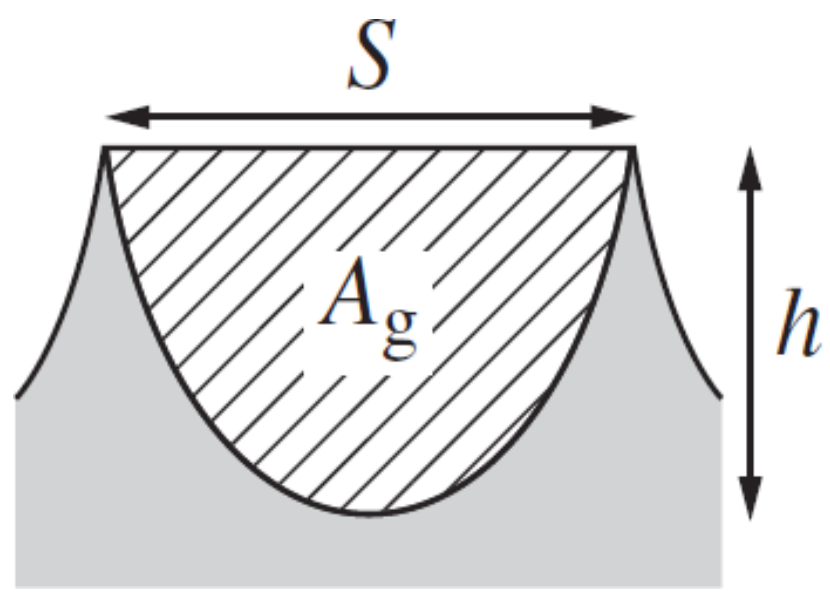

Figure 2. Sketch of groove cross section $A_{g}$. Adapted from Garcia-Mayoral and Jimenez. ${ }^{4}$

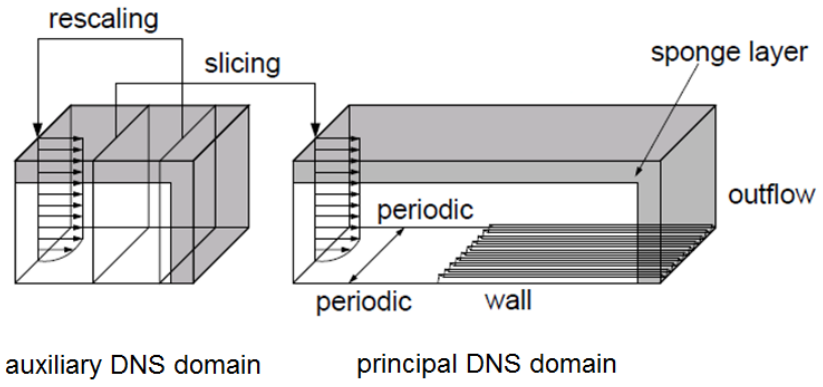

Figure 3. Sketch of DNS domain for spatial simulations. 


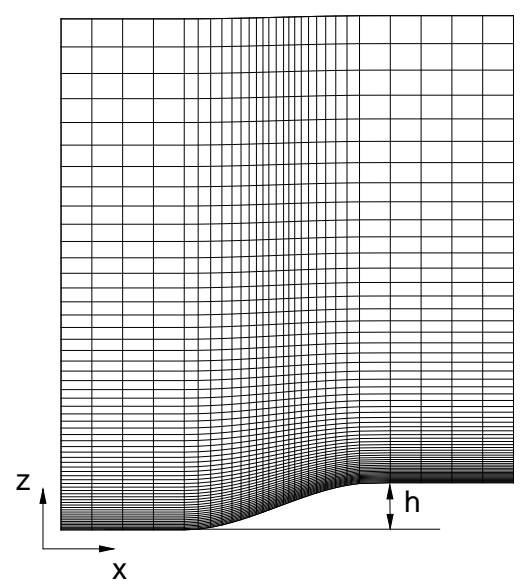

(a) Side view of the transition from clean surface to riblets

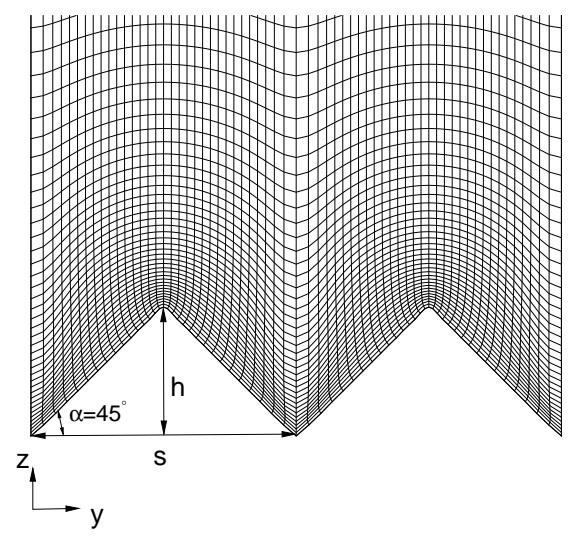

(b) Close-up view of riblet configuration

Figure 4. Computational mesh near riblets for M25s20.

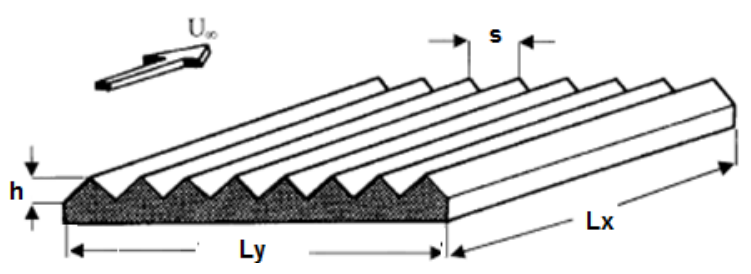

Figure 5. Sketch of riblet geometry. Adapted from Robert. ${ }^{34}$

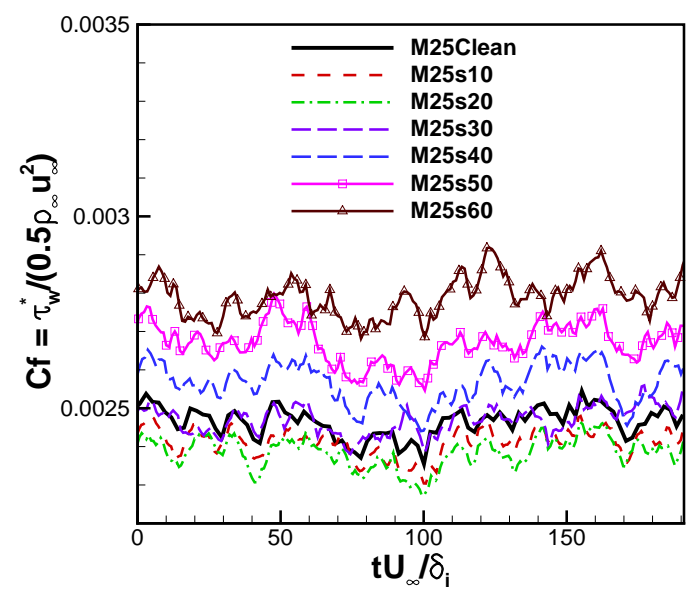

Figure 6. Time history of the skin-friction coefficient for various Mach 2.5 cases. 


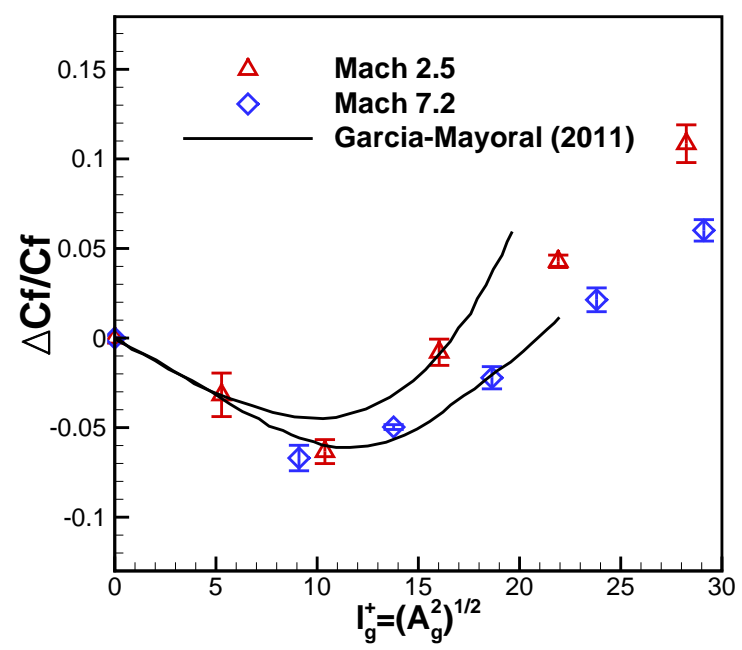

Figure 7. Drag-reduction results from DNS of supersonic flat-plate boundary layers with triangular riblets. The symbols represent DNS results with the error bars estimated based on the variability in drag reduction as a function of the size of the averaging window in $x$ and $t$. The solid lines represent the envelope of the experimental data at low speeds compiled by Garcia-Mayoral and Jimenez. ${ }^{4}$

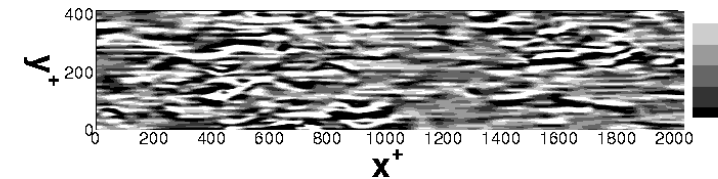

(a) M25s20

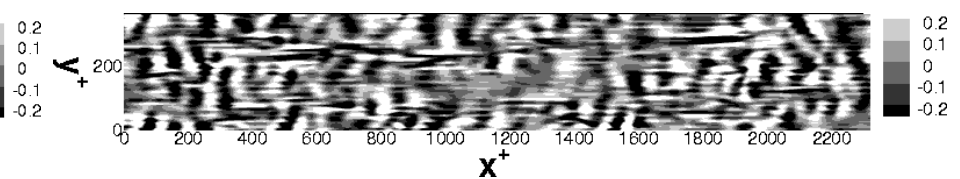

(b) M72s20

Figure 8. Instantaneous wall-normal velocity at $\left(z-z_{t i p}\right)^{+} \approx 3$ within the riblet zone of the simulation domain. The origin in $\mathrm{x}$ has been shifted arbitrarily.

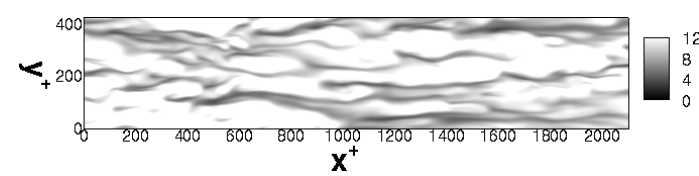

(a) M25Flat

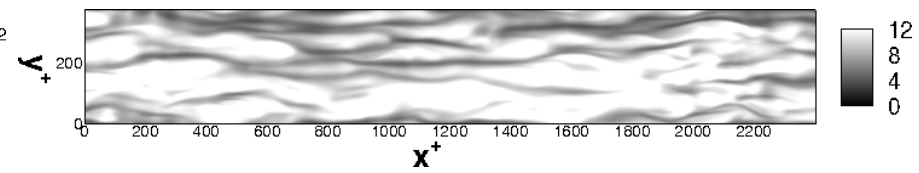

(b) M72Flat

Figure 9. Instantaneous streamwise velocity at $z^{+} \approx 15$ for M25Flat and M72Flat. The origin in $\mathrm{x}$ has been shifted arbitrarily. 


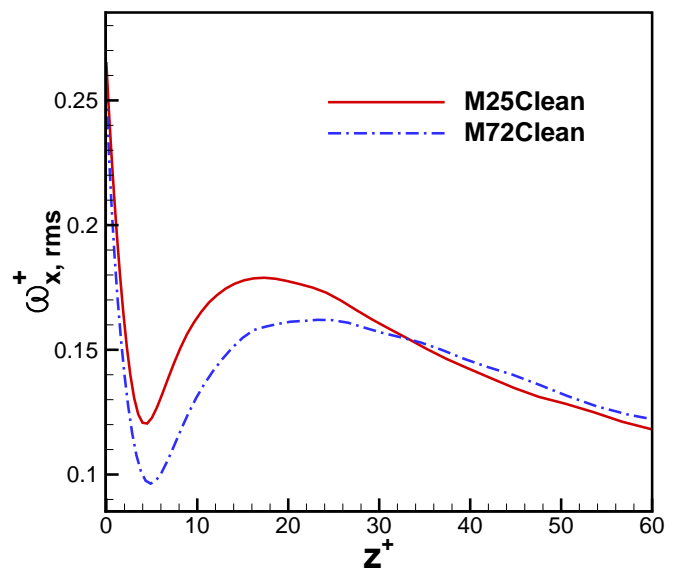

Figure 10. The RMS of streamwise vorticity as a function of the distance away from wall for M25Clean and M72Clean.

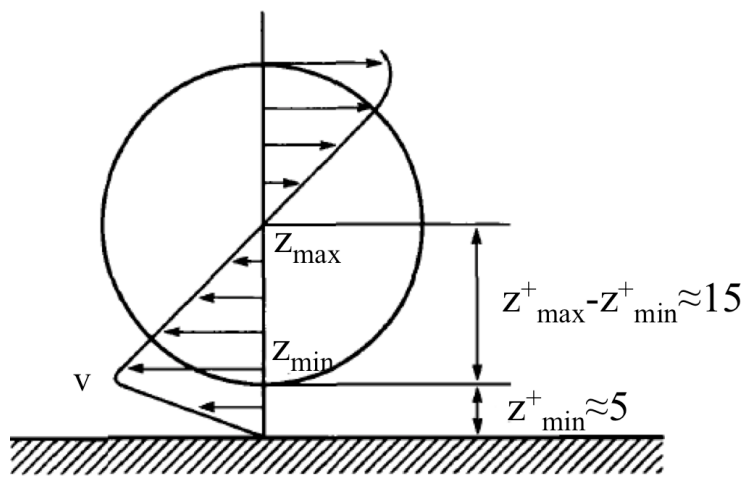

Figure 11. A near-wall streamwise vortex model (Adapted and modified from Kim et al. ${ }^{28}$ ). In the figure, $z_{\max }$ and $z_{\min }$ correspond to the local minimum and local maximum of the rms of streamwise vorticity shown in Figure 10.

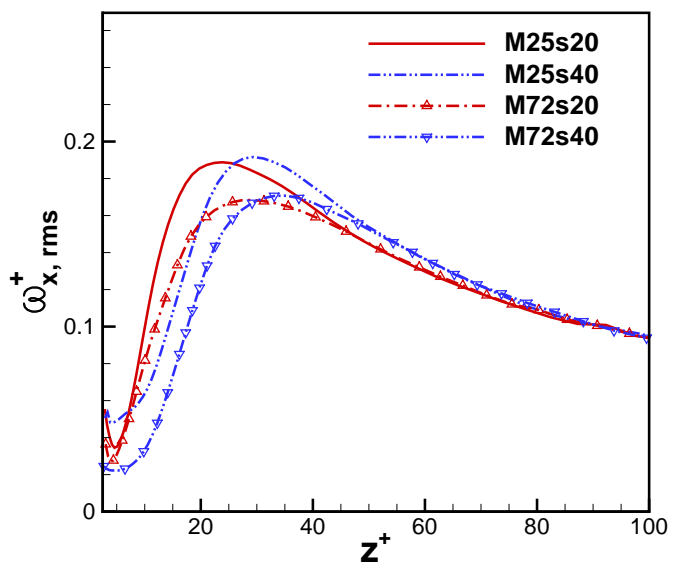

Figure 12. The RMS of streamwise vorticity as a function of wall-normal distance above the riblet valley for several riblet cases. 


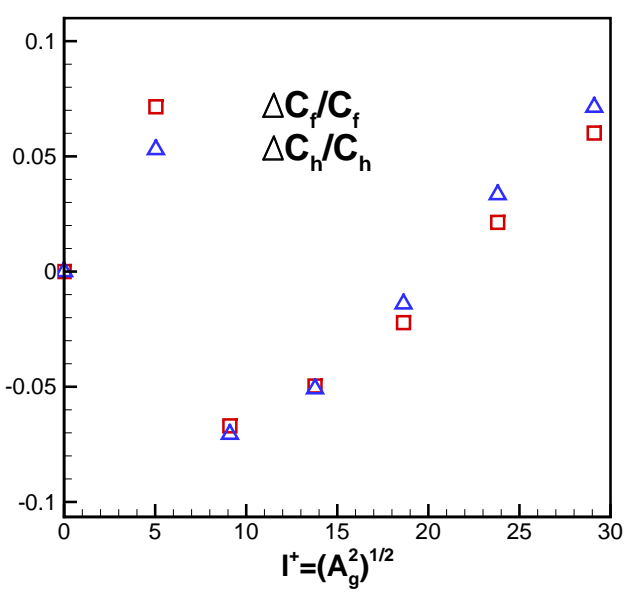

(a)

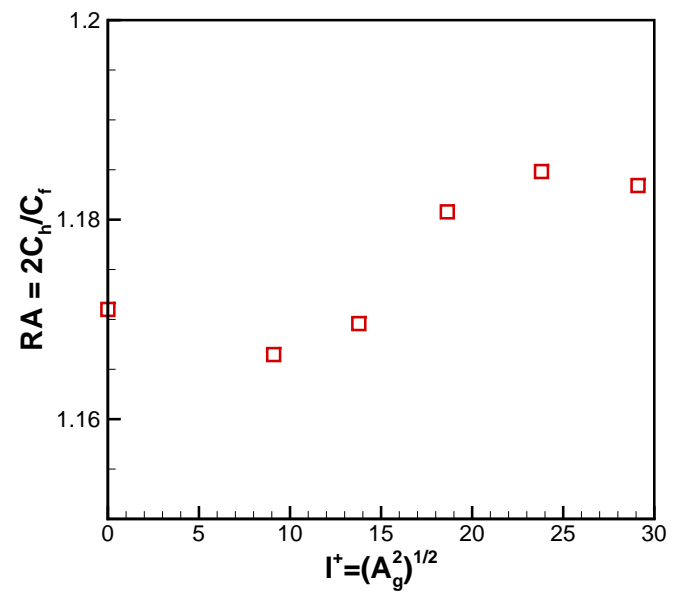

(b)

Figure 13. Stanton number and Reynolds analogy factor as a function of riblet size $l_{g}^{+}$for the Mach 7.2 case with $T_{w} / T_{r}=0.5$. 